\title{
Neue Entwicklungen in der endovenösen Therapie der Varikose
}

In der Therapie insuffizienter Stammvenen gewinnt die endovenöse thermische Ablation zunehmend an Bedeutung. $\mathrm{Zu}$ den neueren Entwicklungen zählt die endovenöse Therapie mit dem Radiallaser, berichtete Dr. Jürgen Böhme, Gefäßchirurg in Lüneburg und Hamburg. Heute haben die hierfür eingesetzten Diodenlaser eine Wellenlänge von $1.470 \mathrm{~nm}$. Entscheidender technischer Fortschritt gegenüber früheren Lasersystemen ist, dass die Radiallaser die Laserenergie ringförmig um die Katheterspitze direkt in die Venenwand emittiert. Zwischenzeitlich stehen Radiallaser mit zwei Ringen zur Verfügung. Damit lässt es sich sehr präzise arbeiten und Schmerzen und Einblutungen sind deutlich reduziert, so Böhme. Sicherheit und Effektivität sind durch Studiendaten belegt [1].

Das Risiko einer Rekanalisation nach thermischer Ablation wird jedoch durch den Durchmesser im Crossenbereich beeinflusst [2]. Böhme bevorzugt bei einem Durchmesser über $10 \mathrm{~mm}$ den Radiallaser, der in dieser Situation einer Radiofrequenz-Ablation überlegen ist [3]. Auch Rezidivbehandlungen führt er mit dem Radiallaser durch. Die üblicherweise verwendete Energie, die das Siebenfache des Venendurchmessers in Joule beträgt, reiche in diesen Fällen jedoch nicht aus. Auf den ersten $4 \mathrm{~cm}$ sollten mindestens $100 \mathrm{~J} / \mathrm{cm}$ appliziert werden. Um einen Stumpf zu vermeiden, positioniert Böhme bei Primäreingriffen die Sondenspitze des Radiallasers an der Vorderwand der tiefen Vena femoralis communis. Bei einem Stumpfrezidiv nach offener Crossektomie muss zunächst der Führungsdraht und die Schleuse in die Vena iliaca externa eingelegt werden, bevor die Katheterspitze an der Vorderwand der tiefen Vene platziert wird. Dies erfordere viel Erfahrung, so Böhme. Zusätzlich erfolgt eine Schaumsklerosierung der kleineren $\ddot{\text { Asste. }}$

Nach der thermischen Ablation wird eine einmalige HeparinProphylaxe gegeben und tägliches Spazierengehen für zweimal 15 Minuten empfohlen bzw., falls dies nicht möglich ist, eine einwöchige Prophylaxe. Erfreulicherweise erstatten immer mehr Krankenkassen die Kosten für endothermale Verfahren, so Böhme.

Angelika Bauer-Delto

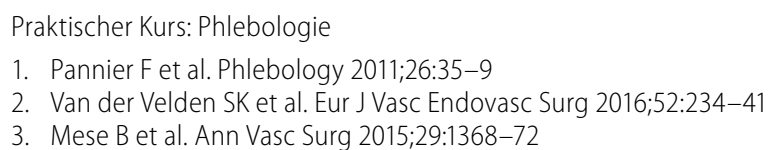

Praktischer Kurs: Phlebologie

2. Van der Velden SK et al. Eur J Vasc Endovasc Surg 2016;52:234-41

. Mese B et al. Ann Vasc Surg 2015;29:1368-72

\section{Update zur Sklerotherapie}

Die aktuelle europäische Leitlinie zur Sklerotherapie der Varikose [1] empfiehlt die Sklerotherapie für alle Formen der Varikose und gibt evidenzbasierte Empfehlungen, die je nach Datenlage in Grad 1A bis 2C eingestuft wurden.

Bei Besenreisern und retikulären Varizen ist die Flüssig-Sklerotherapie Goldstandard (Empfehlungsgrad 1A), eine Sklerosierung mittels Schaum jedoch ebenfalls möglich. Bei größeren Varizen ist die Schaumsklerosierung effektiver. Zu den Indikationen zählen eine inkompetente Vena saphena magna oder par-

Diagnoseverfahren bei chronischen Wunden

Wie der diagnostischen Lücke beim Handekzem begegnet werden kann, ob immer eine Epikutantestung angeraten ist und wann die aktualisierte Leitlinie zum Handekzem veröffentlicht wird, erklärt Dr. Sonja Molin im Video-Interview.

-www.springermedizin.de/link/10555574

AK und PEK: Wann kommt die neue Leitlinie?

Die Leitlinien zur aktinischen Keratose (AK) und zum Plattenepithelkarzinom (PEK) wurden zur Aktualisierung angemeldet, eine Anhebung der Leitlinienstufe ist geplant. Prof. Carola Berking verrät, wann mit der neuen Leitlinie zu rechnen ist

- www.springermedizin.de/link/10560516 va (Empfehlungsgrad 1A), Seitenastvarizen (1B), inkompetente Perforansvenen (1B), eine periulzeröse Varikose (1B) und venöse Malformationen (1B). Ein häufiges Einsatzgebiet der Schaumsklerosierung sind auch Rest- und Rezidivvarizen (1B) nach operativen Eingriffen, berichtete Dr. Franz Xaver Breu, RottachEgern. Die Schaumsklerosierung sei eine gute minimalinvasive Alternative zu chirurgischen Verfahren und mit Abstand die kosteneffektivste Methode der Varizenbehandlung. Sie kann ohne Anästhesie durchgeführt und gut mit anderen Methoden kombiniert werden. Der Patient ist danach sofort wieder arbeitsfähig. Unter Beachtung der Kontraindikationen ist die Sklerotherapie sicher.

Breu gab praktische Tipps, die bei der Durchführung der Schaumsklerosierung zu beachten sind: Pro Sitzung sollten maximal $10 \mathrm{ml}$ Schaum verwendet werden. Dieser sollte möglichst viskös, feinblasig und homogen sein und unter Ultraschallkontrolle verteilt werden. Das behandelte Bein sollte für 2-5 Minuten nicht bewegt werden. Eine sofortige Kompression der injizierten Bereiche sollte vermieden werden. In der Nachbehandlung wird für 24 Stunden ein Kompressionsverband angelegt. Breu empfiehlt, danach für zwei Wochen tagsüber Kompressionsstrümpfe zu tragen. Die Auffassungen, ob und wie lange eine Kompression erfolgen sollte, seien international jedoch nicht einheitlich. Eine Thromboseprophylaxe mit Heparin für eine Woche verordnet Breu in der Regel nur bei erhöhtem Risiko.

Der Patient sei vorab über mögliche Nebenwirkungen und Komplikationen aufzuklären. Zu Hyperpigmentierungen beispielsweise kommt es bei etwa $20 \%$. Diese sind jedoch in der Regel selbstlimitierend und bilden sich nach Wochen bis Monaten wieder zurück.

Angelika Bauer-Delto

Praktischer Kurs: Phlebologie

1. Rabe E et al. Phlebology 2014;29:338-54 